\title{
Invited Commentary: "Event-based versus process-based informed consent to address scientific evidence and uncertainties in ionising medical imaging" by Recchia et al.
}

\author{
Peter Vock
}

Received: 19 July 2013 / Accepted: 12 August 2013 / Published online: 10 September 2013

(C) The Author(s) 2013. This article is published with open access at Springerlink.com

The authors of this article [1] have to be congratulated for their clear presentation of stepwise, process-based informed consent, including evidence-based justification, full patient information on benefits and risks, and interactive communication with enough time for the patient before taking the decision on whether to undergo an examination. Recchia et al. are in line with the clinical consent process [2], and even some radiological articles head in the same direction [3, 4]. The authors will likely get unanimous approval for their approach whenever risky interventions or complex examinations with an elevated dose level are considered. Non-invasive imaging examinations using low-dose levels, however, are not discussed differently by the authors, although this contrasts to the reality in radiology departments in Europe, the USA [5] and probably most countries. In other words, the theory is not applied in a large segment of the real world, and this brings up several questions:

\section{Who is responsible for obtaining informed consent?}

Recchia et al. do not specify this point; they seem to rely on a model where the physician - as a general practitioner-has repeated contacts to the patient, allowing for an ongoing interaction and a chronological separation of the different steps. Most diagnostic radiologists performing radiography, fluoroscopy and CT see their patients just once, for the examination. To achieve a correct informed consent procedure, they either have to offer an additional previous consultation or to share the task with another medical professional, such as the

A reply to this comment is available at doi 10.1007/s13244-013-0284-2

P. Vock $(\square)$

University of Bern, Ahornweg 58, 3095 Spiegel, Switzerland

e-mail: peter.vock@med.unibe.ch referring physician [6]. Correct justification and full information of the patient in the latter scenario are the primary responsibility of the referrer whereas the radiologist acts on demand in the first phase and has the main responsibility in giving answers to questions of the patient, in verifying that the patient has understood the information and in documenting the consent. Whatever the solution, the changes in practice will have a substantial impact on the operational load in an imaging department [6]. This may be the reason for US academic departments to delegate the duty of obtaining informed consent to radiological technologists [5], a solution that probably cannot fully satisfy all needs. In any case, both the referrer, the radiologist and any medical professional involved need adequate education and training in this task. The new guidance document of the European Union for training and education in medical radiation protection, as currently developed in the EC MEDRAPET project, will cover this in detail (www. medrapet.eu).

\section{Is informed consent identical for all imaging examinations} using ionising radiation?

In their clear, interactive approach, Recchia et al. do not differentiate between paediatric whole-body PET-CT and two-plane radiography of a broken finger in an elderly patient. Indeed, the risk of cancer induction may differ by a factor well above 1,000 between these two examples, and it seems logical that our efforts concentrate on those exposure situations with a significant risk. The widespread lack of qualified professional staff and economic constraints of the healthcare systems, due to the financial crisis, underline this point. Thus, we might postulate an approach adapted to the cancer risk, i.e. based on organ doses, the age at exposure and gender. This would mean a 
careful, stepwise process including the parents in the case of children and an abbreviated procedure in the case of elderly patients. Furthermore, imaging in emergency situations has to respect the medical urgency, and situations where the patient is not able to give an informed consent require a modified approach, e.g. after loss of consciousness.

\section{Is there a threshold for the need for risk information?}

Recchia et al. briefly mention authors discussing a minimal effective dose of $1 \mathrm{mSv}$ for detailed risk information [6]. To control the workload in a busy department, this might be an attractive pragmatic option: most radiographic examinations would pass below this threshold, and even modern low-dose $\mathrm{CT}$ protocols might fit, whereas most $\mathrm{CT}$, fluoroscopic and interventional examinations would deserve the full processbased steps for getting informed consent. For higher dose investigations, e.g. those above $10 \mathrm{mSv}$, Malone et al. suggest a more detailed informed consent procedure and forms specific for the examination [6].

Scientific arguments would even favour a risk-based rather than dose-based threshold, e.g. to allow for different decisions for a baby girl and an adult, according to the BEIR VII data [7]. When patient groups were asked about the information they wanted to receive prior to a medical act with potentially severe side effects, Barnett et al. [8] reported a $0.1 \%$ risk to be critical for many; subjective needs for information varied widely and were greater in young than elderly people. Terry mentions that there is no formally recognised threshold of risk above which we ought to obtain informed consent but that an ethical guideline might be any risk greater than that which we might ordinarily encounter when carrying out normal daily activities [2]. This would correspond roughly to a risk of $1: 2,000$

\section{How do we communicate the risk and stochastic uncertainties?}

In a survey of physicians affiliated with tertiary hospitals, Karsli et al. [4] obtained the best support for the following two statements: (1) that X-rays are a known human carcinogen and that there is an epidemiological association between $\mathrm{X}$ rays and cancer; (2) that the risk of cancer from radiation exposure from a CT scan is much smaller than the high background risk of cancer in the population. Recchia et al. [1] are right in suggesting a combination of visual displays and descriptions of the risk in order to help the patient understand the magnitude. It may be easier to perceive the approximate exposure of a specific examination if the dose is expressed by the corresponding number of chest radiographs rather than by the physical dose. Similarly, patients are more likely to get an idea of the risk when this is expressed as "one patient out of $x$ patients" rather than a percent number.

Regarding stochastic risk uncertainties, Recchia et al. clearly state that "patients must be fully informed of what is known and what is unknown". This task is not as easy as they claim and rather time-consuming: while the mean risk to the population by a certain examination can usually be quantified, the individual risk of cancer induction depends on many factors, such as organ dose, sex and age; recent research has even shown that there is an important genetic variation in the sensitivity to ionising radiation. Thus, even if we fully accept the linear no-threshold (LNT) model, it is not easy for experts and difficult for patients to understand the absolute risk to an individual person with its large statistical uncertainty. It may be preferable to justify that the benefits to the patient are well above the range of the risk and that the clinical question cannot be answered by an alternative diagnostic procedure. Arguing with these uncertainties and the difficulty of exactly defining the individual age- and gender-specific risk of an imaging examination, Image Gently and Image Wisely (www.imagegently.org; www.imagewisely.org) do not support a formal informed consent for diagnostic ionising radiation. The two campaigns for pragmatic radiation protection in the US advocate for providing every patient with educational material and for replacing informed consent by a standardised "radiation consent" form, similar to the form used before the injection of contrast media.

In conclusion, there are no easy answers to these questions that do not provoke debates. We need to improve informed consent for radiation exposure in imaging. In times of limited resources, concentrating on critical situations and adapting the process to the magnitude of the risk deserve consideration and might have a stronger impact on the practice of imaging than asking for a rigid full process.

Conflicts of interest The author declares no conflicts of interest. No funding was received for this work.

Open Access This article is distributed under the terms of the Creative Commons Attribution License which permits any use, distribution, and reproduction in any medium, provided the original author(s) and the source are credited.

\section{References}

1. Recchia V, Dodaro A, Braga L (2013) Event-based versus processbased informed consent to address scientific evidence and uncertainties in ionizing medical imaging. Insights Imaging. doi:10.1007/ s13244-013-0272-6

2. Terry PB (2007) Informed consent in clinical medicine. Chest 131:563-568

3. Gunderman RB, Kakarala B (2012) Informed consent in professionalism. AJR 198:W415-W416 
4. Karsli T, Kalra MK, Self JL, Rosenfeld JA, Butler S, Simoneaux S (2009) What physicians think about the need for informed consent for communicating the risk of cancer from low-dose radiation. Pediatr Radiol 39:917-925

5. Lee CI, Flaster HV, Haims AH, Monico EP, Forman HP (2006) Diagnostic CT scans: institutional informed consent guidelines and practices at academic medical centers. AJR 187:282-287

6. Malone J, Guleria R, Craven C, Horton P, Järvinen H, Mayo J, O’Reilly G, Picano E, Remedios D, Le Heron J, Rehani M,
Holmberg O, Czarwinski R (2012) Justification of diagnostic medical exposures: some practical issues. Report of an International Atomic Energy Agency Consultation. Brit J Radiol 85:523-538

7. Health risks from exposure to low levels of radiation: BEIR VII phase 2 (2006) http://www.nap.edu/catalog.php?record_id=11340). Accessed 20 Jul 2013

8. Barnett GC, Charman SC, Sizer B, Murray PA (2004) Information given to patients about adverse effects of radiotherapy: a survey of patients' views. Clin Oncol 16:479-484 